\title{
COMPARISON OF BAYESIAN AND OTHER APPROACHES TO THE ESTIMATION OF FATIGUE CRACK GROWTH RATE FROM 2D TEXTURAL FEATURES
}

\author{
Matej Mojzeš, Jaromír Kukal \\ Czech Technical University in Prague, Faculty of Nuclear Sciences and Physical Engineering, Department of Software \\ Engineering, Praque, Czech Republic \\ e-mail: mojzemat@fjfi.cvut.cz \\ HYNEK LAUSCHMANN \\ Czech Technical University in Prague, Faculty of Nuclear Sciences and Physical Engineering, Department of Materials, \\ Praque, Czech Republic
}

\begin{abstract}
The fatigue crack growth rate can be explained using features of the surface of a structure. Among other methods, linear regression can be used to explain crack growth velocity. Nonlinear transformations of fracture surface texture features may be useful as explanatory variables. Nonetheless, the number of derived explanatory variables increases very quickly, and it is very important to select only few of the best performing ones and prevent overfitting at the same time. To perform selection of the explanatory variables, it is necessary to assess quality of the given sub-model. We use fractographic data to study performance of different information criteria and statistical tests as means of the sub-model quality measurement. Furthermore, to address overfitting, we provide recommendations based on a cross-validation analysis. Among other conclusions, we suggest the Bayesian Information Criterion, which favours sub-models fitting the data considerably well and does not lose the capability to generalize at the same time.
\end{abstract}

Keywords: quantitative fractography, optimization, heuristic, linear regression, sub-model selection

\section{Introduction}

One of the tasks of quantitative fractography consists of modelling the relation between the fatigue crack growth rate (CGR) and textural features of images of fatigue fracture surfaces as explained in (Lauschmann and Siska, 2012; Nadbal et al., 2008; Lauschmann et al., 2006). For this purpose, e.g. a multilinear regression model (Sekeresova and Lauschmann, 2008; Kunz et al., 2010) or a neural network may be used. Of these two, the neural network allows us to analyze and describe the structure of the obtained modeland better imagine the textural subset which is mutually related with the CGR (Lauschmann and Goldsmith, 2009).

The parameters of the respective regression model may be estimated using the least squares method. However, in real-world applications, the basic linear model is not flexible enough to fit the data. This can be solved by adding terms defined by non-linear functions of the basic features, e.g. logarithm, square root, etc. However, adding such features is soon limited by the given number of images.

According to Lauschmann and Goldsmith (2009), one possible way around this limitation is a two-phase stepwise regression with the first stage being bottom-up stepwise regression beginning with a constant model and terminating at a given overfitting level $p_{0}$. In each iteration, a new explanatory variable is included - the one which maximally decreases the sum of squares of residui. The second stage is top-down stepwise regression beginning with the final sub-model 
from the first stage and terminating at the given final overfitting level $p_{F}$. In this procedure, an explanatory variable is selected for the elimination via the Wald test on a selected critical level.

While keeping in mind the relevant motivation for this problem, we suggest that instead of the stepwise regression, an alternative statistical approach based on the method of sub-model multiple testing may provide better results (Mojzeš et al., 2012). There is a vast set of possible criteria that evaluate the quality of a given sub-model and are to be minimized. Further in the paper, we elaborate on the selection and assessment of some of the criteria. They are are interesting in the fractographic context, but may be applied generally to multi-parametric recognition as well.

\section{Material and methods}

\subsection{Linear model}

Denote by $v_{j}$ the crack growth rate assigned to the $j$-th image of the fracture surface, and by $f_{u j}$ the set of image features. The multilinear model in its basic form is based on the formula

$$
\log _{10} v_{j} \approx c_{0}+\sum_{u} c_{u} f_{u j}
$$

Parameters $c_{u}$ can be estimated by the least squares method. Since the linear model is not flexible enough to fit the data, we may add different non-linear functions of basic features and, therefore, modify the model to the following form

$$
\log _{10} v_{j} \approx c_{0}+\sum_{q} c_{q} h_{q}
$$

where $h$ are selected from an extended set of features containing the features $f_{u}$ and a selection of their basic non-linear functions, e.g.

$$
\left\{h_{v}\right\} \subset \mathbf{P} \cup \mathbf{Q} \cup \mathbf{R}
$$

where

$$
\mathbf{P}=\left\{f_{u}\right\} \quad \mathbf{Q}=\left\{\log _{10} f_{u}, f_{u}^{-1}, f_{u}^{1 / 2}, f_{u}^{2}\right\}, \quad \mathbf{R}=\left\{F_{u v}, F_{u v}^{-1}, F_{u v}^{1 / 2}, F_{u v}^{2} \mid u>v\right\}
$$

where $F_{u v}=f_{u} f_{v}$.

The next task will consist of defining a specific methodology on how to select and assess a distinct combination of explanatory variables, i.e. how to select the best sub-model from the extended feature set.

\subsection{Sub-model selection}

A sub-model should be considered a nested subset of the full model consisting of all the variables from the extended feature set. There are two extreme cases: the first is the full model and the second one corresponds to the constant model.

Let $n \in \mathbb{N}$ be the length of $v$ (i.e. the number of observations), $m \in \mathbb{N}$ the extended feature set cardinality and $k \in\{0,1, \ldots, m\}$ the number of explanatory extended feature set variables used in the sub-model. Also, let $\mathbf{c}=\left[c_{0}, c_{1}, \ldots, c_{k}\right]$ be the vector representing sub-model coefficients calculated solving Eq. (2.2). This vector can be divided into $\mathbf{c}_{r e d}=\left[c_{1}, c_{2}, \ldots, c_{k}\right]$ as its significant part and $\mathbf{c}_{0}=\left[c_{0}\right]$ as the constant term coefficient.

Furthermore, we may express the sum of squares for the optimum $\mathbf{c}$ of a given sub-model as $S S Q$ and $S S Q_{0}$ as the sum of squares for $\mathbf{c}_{0}$. Lastly, we will use the sub-model error defined as

$$
s_{e}^{2}=\frac{S S Q}{n-k-1}
$$




\subsection{Data description}

The methods developed in this paper will be applied to fatigue fracture surfaces of three laboratory specimens of the heat-resistant steel P92. Compact tension specimens (Fig. 1) (Lauschmann et al., 2011) were loaded in air at $20^{\circ} \mathrm{C}$ by constant sinusoidal cycles with parameters of the external force according to Table 1. The loading frequency was lowered in steps from $13 \mathrm{~Hz}$ to $4 \mathrm{~Hz}$ in the final stage.

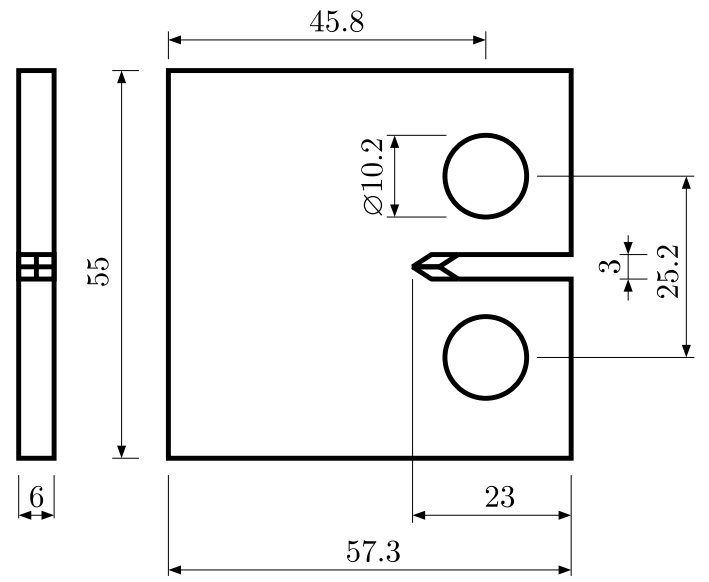

Fig. 1. Compact tension specimen

Table 1. External force parameters

\begin{tabular}{|c|c|c|}
\hline Specimen & $F_{\min }[\mathrm{N}]$ & $F_{\max }[\mathrm{N}]$ \\
\hline \hline 1 & 140 & 3300 \\
\hline 2 & 2000 & 4800 \\
\hline 3 & 3300 & 5500 \\
\hline
\end{tabular}

The fatigue crack surfaces were documented using SEM with magnification $200 \times$, real field of view was $0.6 \mathrm{~mm} \times 0.45 \mathrm{~mm}$ (examples in Fig. 2). The sequence of images was located in the middle of the crack surface along the same axis according to which the crack length was measured (Fig. 3). The recorded areas were mutually shifted by $0.4 \mathrm{~mm}$. The direction of crack growth in the images was bottom-up. Digital representation in $1200 \times 1600$ pixels and 256 brightness values was used. The estimates of CGR were computed from frequently repeated records of the crack length. The course of the CGR related to the crack length was estimated, and every image was assigned a value of the CGR pertinent to its middle.

(a)

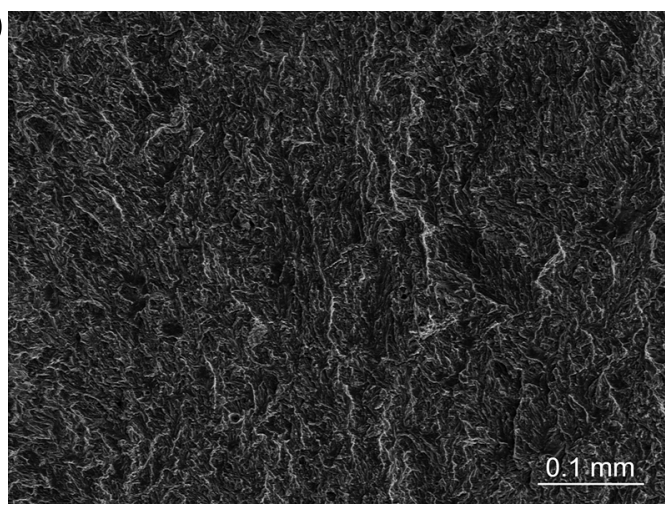

(b)

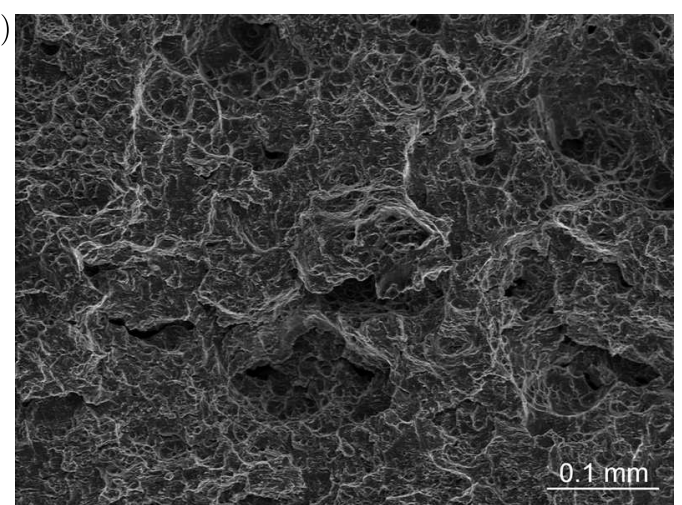

Fig. 2. Examples of SEM pictures of fracture surface; (a) low crack growth rate, (b) high crack growth rate 


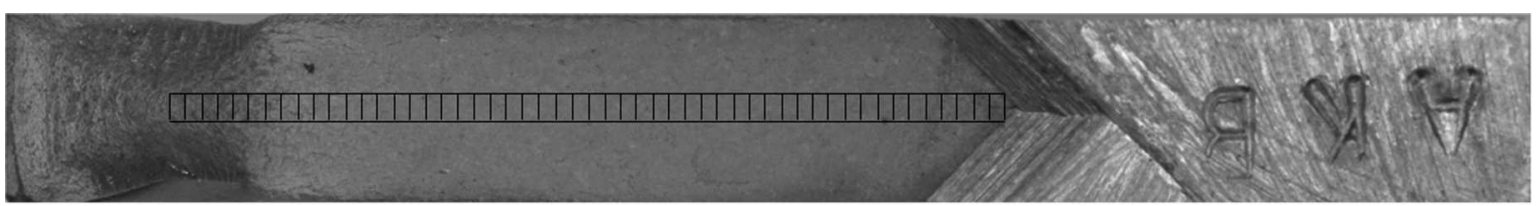

Fatigue crack

Increasing crack growth rate

Fig. 3. Layout of snaps in crack surface (schematic plot)

For image textural features, energies of a 2D discrete wavelet transform were taken (Lauschmann and Goldsmith, 2009). Decomposition using the Type 3 Daubechies wavelet at 8 levels was computed by Matlab function wavedec2. The energy is the mean square of wavelet coefficients for a given level and direction.

The basic sequence of features, $x_{1}, x_{2}, \ldots, x_{24}$, may be regarded as a set of $H_{1}, V_{1}, D_{1}, \ldots, H_{8}, V_{8}, D_{8}$ where $H_{j}, V_{j}, D_{j}$ are wavelet decomposition energies at the $j$-th level in the horizontal, vertical and diagonal directions. The vector $y$ represents decimal logarithm of the crack growth rate $y=\log _{10} v$.

The analysed data consisted of $n=162$ observations and a total of 1224 features in the expanded feature set. It comprises:

- basic linear features $\mathbf{P}, \operatorname{card}(\mathbf{P})=24$,

- non-linear transformations of the basic features $\mathbf{Q}$, $\operatorname{card}(\mathbf{Q})=96$,

- dot product transformations of the basic features $\mathbf{R}, \operatorname{card}(\mathbf{R})=1104$,

as stated in (2.3).

To minimize potential numerical errors when working with the data, input data standardization was implemented as follows

$$
x_{k}=\frac{h_{k}-\mathrm{E} h}{\sqrt{\mathrm{D} h}}
$$

using $\mathrm{E} h$ and $\mathrm{D} h$ as the mean value and dispersion of the explanatory data.

Last, but not least - apart from the significance of the data, we can make use also of physical distribution of the data in the given data set, which is divided randomly into three separate groups. This will be especially useful when dealing with the cross-validation.

\subsection{Selection heuristic}

Searching for the best available sub-model is a binary optimization task that can be defined as minimization of the objective function $f: \mathbf{D} \rightarrow \mathbb{R}$ where

$$
\mathbf{D}=\left\{\mathbf{x} \in\{0,1\}^{m} \mid \mathbf{0} \leqslant \mathbf{x} \leqslant \mathbf{1}\right\}
$$

is the binary domain. Here, the binary vector $\mathbf{x}$ is directly representing utilization of the extended feature set, i.e. its components that are equal to "one" are included in the corresponding sub-model. Therefore, $\mathbf{0}$ refers to the constant model, and $\mathbf{1}$ to the full model.

Furthermore, suppose that we have an acceptable value of the objective function $f^{*}$. Then, we can define a set of solutions, the goal set, as

$$
\mathbf{G}=\left\{\mathbf{x} \in \mathbf{D} \mid f(\mathbf{x}) \leqslant f^{*}\right\}
$$

where

$$
f^{*} \geqslant \min \{f(\mathbf{x}) \mid \mathbf{x} \in \mathbf{D}\}
$$


For that purpose, we may utilize some of the well-known heuristic algorithms. We have chosen physically motivated Fast Simulated Annealing (FSA) (Kvasnička et al., 2000) with reputable efficiency in the case of integer optimization tasks. FSA performs mutation on the ring neighbourhood

$$
N(\mathbf{x})=\left\{\mathbf{y} \in \mathbf{D} \mid\|\mathbf{y}-\mathbf{x}\|_{1}=1\right\}
$$

Beginning with $k=0, T_{k}>0$ and the initial solution vector generated by uniform distribution $\mathbf{x}_{0} \sim U(\mathbf{D})$, we perform FSA mutation as a uniformly generated random binary vector $\mathbf{y}_{k} \sim U\left(N\left(\mathbf{x}_{k}\right)\right)$. Using $\eta_{k} \sim U([-1,+1])$, we set

$$
\mathbf{x}_{k+1}=\left\{\begin{array}{lll}
\mathbf{y}_{k} & \text { for } & f\left(\mathbf{y}_{k}\right)<f\left(\mathbf{x}_{k}\right)+T_{k} \tan \frac{\pi \eta}{2} \\
\mathbf{x}_{k} & \text { for } & f\left(\mathbf{y}_{k}\right) \geqslant f\left(\mathbf{x}_{k}\right)+T_{k} \tan \frac{\pi \eta}{2}
\end{array}\right.
$$

until a solution from the goal set is found, or the pre-defined number of objective function evaluations is exhausted. The cooling strategy is represented by a non-increasing sequence of positive temperatures $T_{k}$.

We have been inspired by the increased efficiency of hybrid heuristics in the case of combination of a differential evolution and the steepest descent (Tvrdík J and Křivý, 2011). And since the previously defined set of optimization problems has many local minima, we have enhanced the FSA algorithm by a hybrid part - the steepest descent, which may increase the probability of reaching the global optimum.

In our approach to hybrid heuristic optimization, instead of $f(\mathbf{x})$ optimization, we optimize $g(\mathbf{x})=f(\mathbf{h})$ where $\mathbf{x}=\mathbf{x}_{0}, \mathbf{h}=\mathbf{x}_{H}$ are the first and last members of any series $\left\{\mathbf{x}_{k}\right\}_{k=0}^{H}$ satisfying $\mathbf{x}_{j} \in N\left(\mathbf{x}_{j-1}\right), f\left(\mathbf{x}_{j}\right)<f\left(\mathbf{x}_{j-1}\right)$ for $j=1, \ldots, H$. This means $\mathbf{h}$ is the best solution in terms of the steepest descent heuristic. Before any problem solution vector is evaluated, its nearest local neighbourhood is iteratively searched for a better solution until no further advance in terms of the objective function can be made (or until a pre-defined maximum number of local evaluations is exceeded).

This way we were able to set a higher temperature $T_{0}$ and use a more benevolent cooling strategy. In other words, the algorithm is able to prevent getting stuck in a local minimum and still not lose the ability to fine-tune a given solution. Thus the FSA performance, on this specific task, has been improved.

\subsection{Cross-validation}

Having the data divided into three distinct groups allows us to perform strong cross-validation to assess how the results of a specific criterion will generalize to an independent data set.

We will perform the optimization on two out of three groups (training groups) and validate the analysis on the remaining third group (verification group). To improve the overall consistency, multiple rounds of cross-validation will be performed using different permutations of the data sets, and the verification results will be aggregated over the rounds.

As the goodness of fit measure we propose to use $R$ as the correlation coefficient between the original data and the data proposed by the respective sub-model. However, during the optimization itself, we will use the original objective function based on the minimization of the CRIT value. 


\section{Theory of sub-model selection}

At this point, we should choose some of many possibilities for testing sub-model quality. We have selected a few of them in accordance with (Mojzeš et al., 2016) that can be divided in two sets, based on the concepts they are based on. The first one comprises traditional statistical tests and the criterion that will reflect the quality of a sub-model will be the logarithm of the $p_{\text {value }}$. On the other hand, the second set contains different statistical information criteria regarding selection of the model. In the latter case, we are simply minimizing the value of the selected information criterion.

\subsection{Sub-model testing}

Here, we test significance of a sub-model described by its $\mathbf{c}_{r e d}$. Respective hypotheses are defined as

$$
\begin{array}{ll}
H_{0}: & \mathbf{c}_{r e d}=\mathbf{0} \\
H_{1}: & \mathbf{c}_{r e d} \neq \mathbf{0}
\end{array}
$$

and the $R$-square as well as the Wald test are used for their testing.

$\mathrm{R}$-square test

In order to use $\mathrm{R}^{2}$ for the analysis of the sub-model and constant model, according to the variance analysis (Wooldridge, 2002), we must define the stochastic variable $F$

$$
F=\frac{S S Q_{0}-S S Q}{S S Q} \frac{n-k-1}{k}
$$

Here $F$ belongs to $\mathrm{F}_{k, n-k-1}$ and the respective $p_{\text {value }}=1-\mathrm{F}_{k, n-k-1}(F)$.

Wald test

Alternatively, to test sub-model hypotheses via the Wald test (Anděl, 1978), the variable $Z$ has to be used

$$
Z=\frac{1}{k s_{e}^{2}} \mathbf{c}^{\mathrm{T}} \mathbf{W}^{-1} \mathbf{c}
$$

The matrix $\mathbf{W}$ is a result of $\left(\mathbf{X}^{\mathrm{T}} \mathbf{X}\right)^{-1}$ without the first row and column. Finally, $Z$ has the distribution $\mathrm{F}_{k, n-k-1}$ and $p_{\text {value }}=1-\mathrm{F}_{k, n-k-1}(Z)$.

Lastly, for both tests, the resulting sub-model quality criterion can be defined as

$$
C R I T=\log _{10} p_{\text {value }}
$$

to be minimized. Due to the fact that values of $p_{\text {value }}$ may get very close to zero, it is necessary to avoid potential numerical issues and express $p_{v a l u e}$ in terms of an incomplete gamma distribution.

\subsection{Information criteria}

Another approach to the assessment of the sub-model quality is based on statistical information criteria. The selected criteria are presented in order from the least stringent one. 
Wilks Information Criterion

Ralston and Rabinowitz (2001) according to Wilks (1962) recommend searching for a sub-model having the minimal error $s_{e}^{2}$. The corresponding logarithmic form, consistent with the following criteria, can be defined as

$$
W I C=n \ln s_{e}^{2}
$$

In this basic criterion, $k$ (i.e. the number of explanatory variables included in the sub-model) is already indirectly penalizing the information quality.

\section{Akaike Information Criterion}

Furthermore, an additional penalty for adding more explanatory variables is included in the Akaike criterion measuring relative goodness of the sub-model (Akaike, 1974) denoted as

$$
A I C=W I C+2 k
$$

\section{Bayesian Information Criterion}

Bayesian criterion (Schwarz, 1978) prevents overfitting even more by generating a stronger penalty for extra explanatory variables. Following the existing terminology, the criterion may be defined as

$$
B I C=W I C+k \ln n
$$

for $n \geqslant 8$.

As opposed to the logarithm of $p_{\text {value }}$, the final $C R I T$ to be minimized is directly equal to values of the information criteria.

\section{Results and discussion}

\subsection{Heuristic optimization}

In order to compare the results achieved with the hybrid heuristic to the stepwise alternative, we have implemented a traditional stepwise approach (Mojzeš et al., 2012). Despite using only 125 variables (basic linear and non-linear transformations, $\mathbf{P}$ and $\mathbf{Q}$ ) the heuristic sub-model optimization approach was superior to the stepwise approach based on the best correlation coefficient found.

Table 2 aggregates the best results achieved with the current, much more computationally demanding, data. In multiple runs of the heuristic optimization, we were able to obtain even better values of the correlation coefficient. Still, as the ultimate target should be the ability to generalize the independent data set, we will draw final conclusions based on the following cross-validation.

\subsection{Cross-validation}

The full data set has been divided into three groups of data, each having 59 (Group I), 53 (Group II) and 50 (Group III) observations. For each permutation of training and verification groups the hybrid heuristic optimize the sub-model to make the model fit the training data as well as possible according to the respective method. The same settings and conditions were used as in the case of the full data set without cross-validation. The detailed results are organized 
Table 2. Optimal sub-model quality and features using hybrid heuristic

\begin{tabular}{|c|c|c|c|c|c|c|c|c|c|c|c|c|}
\hline \multirow{2}{*}{ Method } & \multirow{2}{*}{ CRIT } & \multirow{2}{*}{$R$} & \multirow{2}{*}{$k_{o p t}$} & \multicolumn{9}{|c|}{ Term } \\
\hline & & & & $f_{u}$ & $f_{u}^{1 / 2}$ & $f_{u}^{2}$ & $f_{u}^{-1}$ & $\log _{10} f_{u}$ & $F_{u v}$ & $F_{u v}^{1 / 2}$ & $F_{u v}^{2}$ & $F_{u v}^{-1}$ \\
\hline$\overline{F \text {-test }}$ & -117.92 & 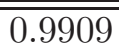 & $\overline{27}$ & 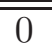 & $\overline{00}$ & 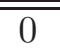 & 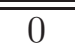 & $\overline{0}$ & $\overline{77}$ & $\overline{4}$ & $\overline{c 11}$ & $\overline{5}$ \\
\hline Wald test & -100.29 & 0.9839 & 15 & 0 & 0 & 0 & 1 & 0 & 1 & 3 & 7 & 3 \\
\hline WIC & -1099.00 & 0.9993 & 88 & 1 & 1 & 4 & 1 & 1 & 14 & 19 & 22 & 25 \\
\hline$\overline{\mathrm{AIC}}$ & -927.03 & 0.9992 & 82 & 0 & 1 & 2 & 1 & 0 & 20 & 19 & 18 & 21 \\
\hline $\mathrm{BIC}$ & -626.86 & 0.9871 & 20 & 0 & 0 & 1 & 0 & 1 & 1 & 2 & 13 & 2 \\
\hline
\end{tabular}

Table 3. Cross-validation results

\begin{tabular}{|l|c|r|c|c|c|}
\hline \multicolumn{1}{|c|}{ Method } & Training groups & CRIT & $R_{\text {train }}$ & $R_{\text {verify }}$ & $k_{\text {opt }}$ \\
\hline \hline $\mathrm{R}^{2}$ test & I+II & -86.72 & 0.9970 & 0.5937 & 34 \\
\hline $\mathrm{R}^{2}$ test & II+III & -76.70 & 0.9900 & 0.5785 & 13 \\
\hline $\mathrm{R}^{2}$ test & I+III & -79.29 & 0.9924 & 0.9594 & 22 \\
\hline Wald test & I+II & -74.29 & 0.9867 & 0.8624 & 10 \\
\hline Wald test & II+III & -71.34 & 0.9848 & 0.6661 & 5 \\
\hline Wald test & I+III & -66.24 & 0.9854 & 0.9476 & 13 \\
\hline WIC & I+II & -736.29 & 0.9991 & 0.4471 & 55 \\
\hline WIC & II+II & -804.83 & 0.9997 & 0.5214 & 61 \\
\hline WIC & I+III & -716.25 & 0.9993 & 0.8286 & 63 \\
\hline AIC & I+II & -619.41 & 0.9988 & 0.8292 & 48 \\
\hline AIC & II+III & -674.31 & 0.9996 & 0.1351 & 56 \\
\hline AIC & I+III & -584.22 & 0.9987 & 0.9229 & 48 \\
\hline BIC & I+II & -479.12 & 0.9984 & 0.6896 & 45 \\
\hline BIC & II+III & -500.70 & 0.9995 & 0.3800 & 53 \\
\hline BIC & I+III & -430.91 & 0.9926 & 0.9574 & 21 \\
\hline
\end{tabular}

in Table 3. The most important results are in the column of the correlation coefficient $R_{\text {verify }}$ which measures the quality of the fit on the verification data set.

These results are aggregated using the mean of respective methods and, furthermore, expanded by comparing the data composed from distinct verification data sets to the original one in Table 4. Also, the results of composed cross-validation are depicted in Fig. 4 as log-log plots of measured and predicted CGRs. The predicted data are distinguished by group symbols $(\times,+, \circ)$. The results of WIC and AIC are the worst ones, since the outliers had to be omitted to enable the plotting. As can be seen, the Wald approach and BIC criterion offer the best results.

Table 4. Cross-validation summary

\begin{tabular}{|l|c|c|}
\hline \hline \multicolumn{1}{|c|}{ Method } & Mean $R$ & Composed $R$ \\
\hline \hline $\mathrm{R}^{2}$ test & 0.7105 & 0.7043 \\
\hline Wald test & 0.8254 & 0.7702 \\
\hline WIC & 0.5990 & 0.5320 \\
\hline AIC & 0.6291 & 0.3281 \\
\hline BIC & 0.6757 & 0.6096 \\
\hline
\end{tabular}


(a)

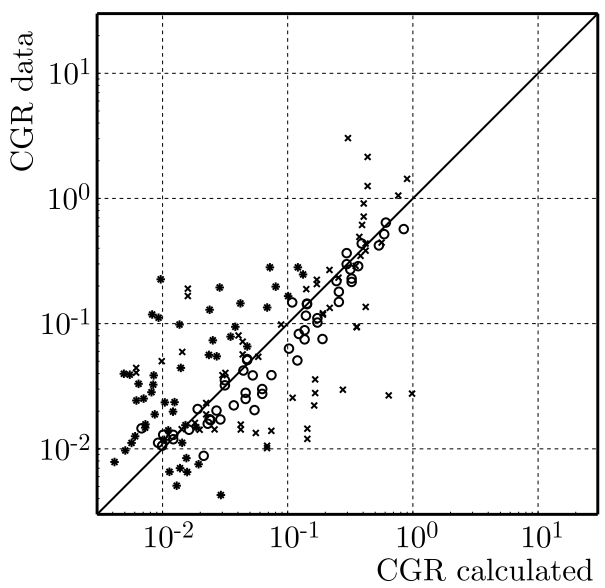

(c)

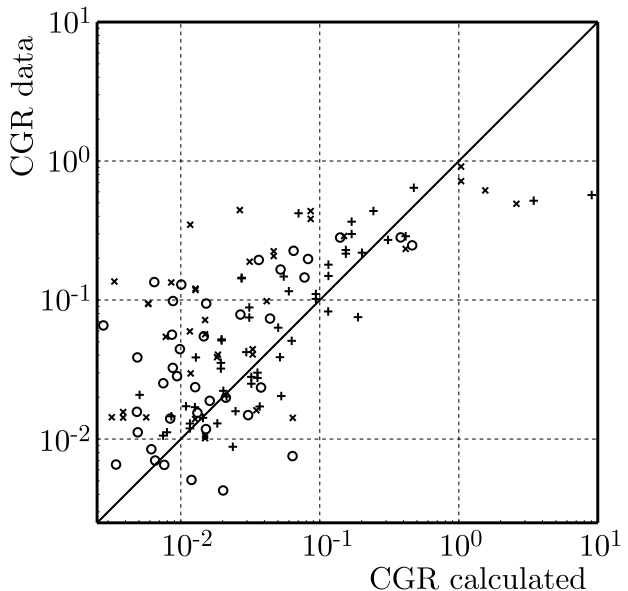

(b)

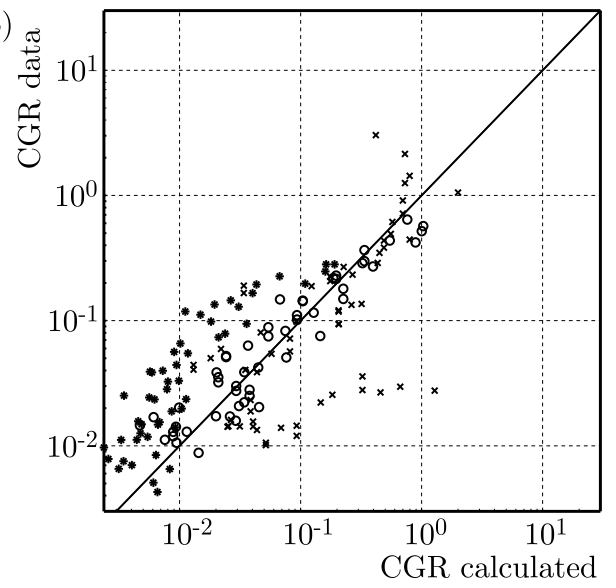

(d)

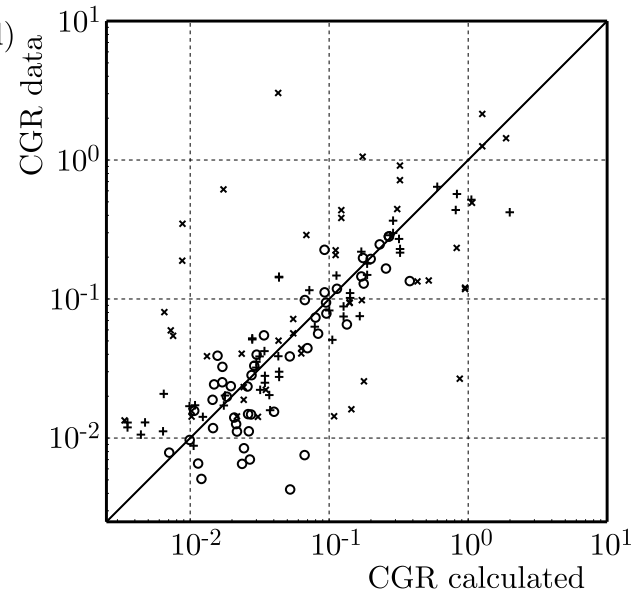

(e)

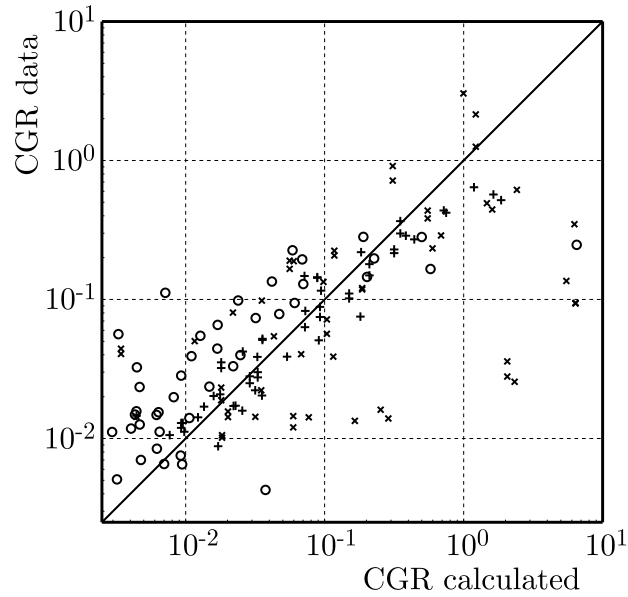

Fig. 4. Prediction of Crack Growth Rate (CGR) using various techniques of sub-model selection $(\times$ representing data in group I, + in group II, and $\circ$ in group III $)$

\section{Conclusions}

The benefits of the solution described above are considerable. Nearly an unlimited set of explanatory variables may be offered without any respect to the original number of observations in a given case. Very good models have been obtained also in previously unsolvable cases with a very small number of observations.

Of course, the final result is mostly dependent on the approach of sub-model selection. As it is apparent from the results of cross-validation, and also based on our experience, we are recommending BIC, Wald test and potentially also $\mathrm{R}^{2}$ test and WIC. Nevertheless, there are significant differences between these four and, more specifically, we are suggesting: 
- BIC as a universal criterion,

- Wald test as a well balanced criterion, similar to BIC,

- $\mathrm{R}^{2}$ test as a legitimate criterion with respect to the variance analysis approach,

- WIC as a criterion that leads to considerable adherence to the data, however, as opposed to other criteria, lacks the ability of generalization.

Acknowledgement

This paper was created under the support of grant OHK4-165/11 CTU in Prague.

\section{References}

1. AKAIKE H., 1974, A new look at the statistical model identification, IEEE Transactions on Automatic Control, AC-19, 716-723

2. AndĚL J., 1978, Mathematical Statistics (in Czech), SNTL/Alfa, Praha

3. Kunz J., Kovarik O., Lauschmann H., Siegl J., Augustin P., 2010, Fractographic reconstitution of fatigue crack growth in integrally stiffened panels, FATIGUE 2010, 2, 1711-1720

4. KvasničKa V., Pospíchal J., TiŇo P., 2000, Evolutionary Algorithms (in Slovak), STU Bratislava

5. Lauschmann H., Goldsmith N., 2009, Textural fractography of fatigue fractures, [In:] Fatigue Crack Growth: Mechanics, Behavior and Prediction, A.F. Lignelli (Edit.), Nova Science Publishers, Inc., 125-166

6. Lauschmann H., Kunz J., KovaŘík O., 2011, Analyzing crack growth rate in steel P92 (in Czech), Research report V-KMAT-839/11, FNSPE CTU Prague

7. Lauschmann H., Siegl J., Sumbera J., Siska F., Nedbal I., 2006, An unifying concept for fatigue: The reference crack growth rate, Materials Characterization, 56, 257-265

8. Lauschmann H., Siska F., 2012, The reference texture: A proposal of a physical explanation, International Journal of Fatigue, 43, 120-127

9. Mojzeš M., Klimt M., Kukal J., 2016, Feature selection via competitive levy flights, 2016 International Joint Conference on Neural Networks (IJCNN)

10. Mojzeš M., Kukal J., Lauschmann H., 2012, Sub-model testing in fractographic analysis, Proceedings of Mendel 2012 Soft Computing Conference, Brno University Technology Press, $350-355$

11. Nedbal I., Lauschmann H., Siegl J., Kunz J., 2008, Fractographic reconstitution of fatigue crack history - Part II, Fatigue and Fracture of Engineering Materials and Structures, 31, 177-183

12. Ralston A., Rabinowitz P., 2001, A First Course in Numerical Analysis, Courier Dover Publications

13. Sekeresova Z., Lauschmann H., 2008, Multi-fractal features of fatigue crack surfaces in relation to crack growth rate, Materials Structure and Micromechanics of Fracture V, 567-568, 129-132

14. Schwarz G., 1978, Estimating the dimension of a model, Annals of Statistics, 6, 461-464

15. Tvrdík J., KŘIvÝ I., 2011, Hybrid adaptive differential evolution in partitional clustering, Proceedings of 17th International Conference on Soft Computing, Mendel 2011, VUT Brno, Brno, $1-8$

16. Wilks S.S., 1962, Mathematical Statistics, John Wiley \& Sons, Inc., New York

17. Wooldridge J.M., 2002, Econometric Analysis of Cross Section and Panel Data, Cambridge, MA: MIT Press 